Original paper

\title{
Health-related quality of life in liver cirrhosis patients using SF-36 and CLDQ questionnaires
}

\author{
Janani K, Mayank Jain, Joy Vargese, Vijaya Srinivasan, Kavya Harika, Tom Michael, Jayanthi Venkataraman \\ Gleneagles Global Health City, Chennai, India
}

\begin{abstract}
Aim of the study: To determine the factors that are likely to influence the domains of health-related quality of life (HRQOL) using SF-36 and CLDQ questionnaires in patients with liver cirrhosis.

Material and methods: Patients with liver cirrhosis were compared with age- and gender-matched healthy controls for physical and mental components of the SF-36 score. Effects of age, co-morbidity, namely diabetes, severity of liver disease and complications of liver cirrhosis on HRQOL using self-administered or by direct interview SF-36 and CLDQ questionnaires were studied. Statistical analysis: chi square test, ANOVA, Kruskal-Wallis test and stepwise linear regression analysis. A $p$ value of $<0.05$ was considered significant.

Results: Regarding SF-36 score, except for bodily pain, 149 patients had significantly low individual and composite domain scores ( $p$ value $<0.0001$ ) compared to age/gender-matched controls. Patients below 45 years, the majority of whom belonged to Child-Turcotte-Pugh (CTP) class C with a high Model of End-Stage Liver Disease (MELD) and higher rates of complication had low SF-36 for bodily pain (KW $p<0.005$ ) and those above 55 years for physical function $(p<0.05)$. Both the physical components had a major impact on mental composite score (MCS) (KW $p<0.05)$. The overall CLDQ score was also low in patients below 45 years old $(p<0.05)$. Diabetes with or without other co-morbid conditions had no effect on SF-36 or CLDQ scores, while non-diabetic co-morbid conditions did on physical domains (physical function, bodily pain and role physical) and the physical component score of SF-36 (KW $p<0.01$ to $<0.0001$ ). In linear regression, MELD had a direct and significant association with overall PCS, MCS and CLDQ.

Conclusions: Age below 45 years, higher MELD and CTP score with the presence of ascites and hepatic encephalopathy affect the overall CLDQ scores.
\end{abstract}

Key words: quality of life, cirrhosis liver.

Address for correspondence

Dr. Mayank Jain, Global Health City, 439 Cheran Nagar, 600100, Chennai, India, e-mail: mayank4670@rediffmail.com

\section{Introduction}

Quality of life (QOL) has attained importance in several gastrointestinal disorders such as gastroesophageal reflux disease, inflammatory bowel disease and chronic liver diseases (CLD) such as cirrhosis. Day-today QOL is influenced not only by the primary disease but also by its management and disease-related complications.

In liver cirrhosis, the severity and progressive nature of the disease [1-3] are likely to have a considerable negative impact on the day-to-day QOL. Manage- ment issues that can also affect QOL include repeated therapeutic paracentesis, endoscopic variceal band ligation for recurrent variceal bleeding, altered sensorium, disturbed sleep pattern and renal dialysis.

The eventual aim of managing patients with liver cirrhosis is not to merely prolong life with poor quality, but to improve and sustain a reasonably good quality of life.

An overall rating of well-being in a patient with liver cirrhosis is conceivable by using a health-related quality of life questionnaire (HRQOL), many of which have been translated and validated in clinical studies $[4,5]$. 
SF-36 is a non-disease (generic) specific questionnaire that provides a composite scale of mental and physical health for patients with any chronic ailment such as gastroesophageal reflux disease, chronic liver disease, inflammatory bowel disease, etc. The domains of SF-36 include general health $(\mathrm{GH})$, physical $(\mathrm{PF})$ and social functioning (SF), bodily pain (BP), role-physical (RP), mental health $(\mathrm{MH})$, role-emotional $(\mathrm{RE})$ and vitality (VT). CLDQ is a disease-specific validated questionnaire for assessment of QOL in patients with chronic liver disease. The questionnaire incorporates disease-specific as well as physical and mental domains and includes 29 items related to QOL that are categorised in 6 domains [6]. The latter includes abdominal symptoms (AS), fatigue (FA), systemic symptoms (SS), activity (AA), emotional function (EF) and worry (WO). The response to the questionnaire is graded on a scale of 1 to 7 and ranges from "all of the time" to "none of the time". This questionnaire is now available in English, Hindi and Tamil and can be used in day-today practice after receiving copyright permission.

The aim of the study was to determine whether factors such as age, co-morbidity with special reference to diabetes mellitus, severity score (Child-Turcotte-Pugh score) and disease-related complications in patients with cirrhosis liver had an impact on individual and composite domains of SF-36 and CLDQ scores.

\section{Material and methods}

This was a case control study with data collection between April 2013 and January 2014. The study group comprised patients (from the Indian subcontinent) with liver cirrhosis who were registered in the liver clinic for either workup (end stage liver disease patients listed for liver transplant) or for management of cirrhosis-related complications. Diagnosis of cirrhosis was based on clinical, biochemical and radiological imaging that included ultrasound and/or contrast-enhanced computed tomography (CECT).

Co-morbidity was categorised as diabetes and non-diabetes related, as a close link between diabetes and the liver is well established [7]. The latter included other co-morbid states such as hypertension, hypothyroidism, arthralgia, psychiatric states, skin ailments like psoriasis etc, which were less likely to have an impact on the liver and were classified as non diabetes co-morbid conditions.

Severity of disease was assessed by Child-TurcottePugh (CTP) score and Model of End-Stage Liver Disease (MELD). Both were calculated on the day of the interview. Concurrent controls were single age/gender matched and selected from hospital employees and medical representatives visiting the hospital during the study period. It was ensured that the controls had no recent illness/hospitalisation/absenteeism due to illness within the preceding 6 months of the study. Presence of comorbid states such as diabetes, hypertension, hypothyroidism and coronary heart disease were not an exclusion criterion for the controls.

The SF-36 questionnaire was used for both cases and controls while the validated CLDQ questionnaire was used for cases only.

Inter-observer variation on 5 sets of English questionnaires was done by VS and VJ with $95 \%$ concurrence. All the investigators were trained and standardised for data collection in both questionnaires. Self-administered questionnaires were completed by patients who were proficient in any one of the 3 languages: English, Hindi or Tamil.

The sample size for the study was computed for patients in CTP A, B and C expecting patients with poor SF-36 and CLDQ scores to provide an odds ratio of 2. The maximum sample size reached for the 2 questionnaires was taken as the sample size for CTP A, B and C in that group of patients.

\section{Exclusion criteria}

Patients who were terminally ill, haemodynamically unstable, and too ill to complete the questionnaire or those with extrahepatic portal hypertension (pre- and post-sinusoidal), i.e. Budd-Chiari syndrome, portal vein thrombosis, non-cirrhotic portal hypertension, were excluded.

The Ethical Committee of the institution approved the study (ref no HR/2013/MS/004). Informed and written consent was obtained from the patients before completing the questionnaire.

Permission was obtained from Younassi et al. for using the English, Hindi and Tamil translated version of CLDQ.

\section{Analysis}

The differences in baseline characteristics that included demography, aetiology, co-morbidity (diabetes mellitus) and cirrhosis associated complications (hepatic encephalopathy, variceal bleeding, refractory ascites, spontaneous bacterial peritonitis, acute kidney injury and hepatocellular carcinoma) belonging to CTP A, B and C were assessed.

For SF-36 and CLDQ questionnaires, individual domains of both SF-36 and CLDQ were assessed. The SF-36 scores were grouped to give composite measures of physical health labelled as physical composite score 
Table 1. Baseline characteristics and SF-36 comparison between cases and controls

\begin{tabular}{lccc}
\hline & Cases & Controls & $\begin{array}{c}\text { Chi square/MW* } \\
p \text { value }\end{array}$ \\
\cline { 1 - 2 } Age $($ mean \pm SD) & $51.6 \pm 9.8$ & $50.8 \pm 8.9$ & NS $^{\#}$ \\
\hline Gender & $126(\mathrm{M}): 23(\mathrm{~F})$ & $126(\mathrm{M}): 23(\mathrm{~F})$ & $\mathrm{NS}^{\#}$ \\
\hline \multicolumn{2}{c}{ Co-morbidity (No. \%) } & $<0.0001$ \\
\hline No co-morbidity & \multicolumn{2}{c}{$68(45.5 \%)$} & \\
\hline Diabetes mellitus with or without other co-morbidity & $76(52.4 \%)$ & $22(15.2 \%)$ & \\
\hline Non diabetes with other co-morbidity & $34(23.4 \%)$ & $57(39.3 \%)$ & \\
\hline
\end{tabular}

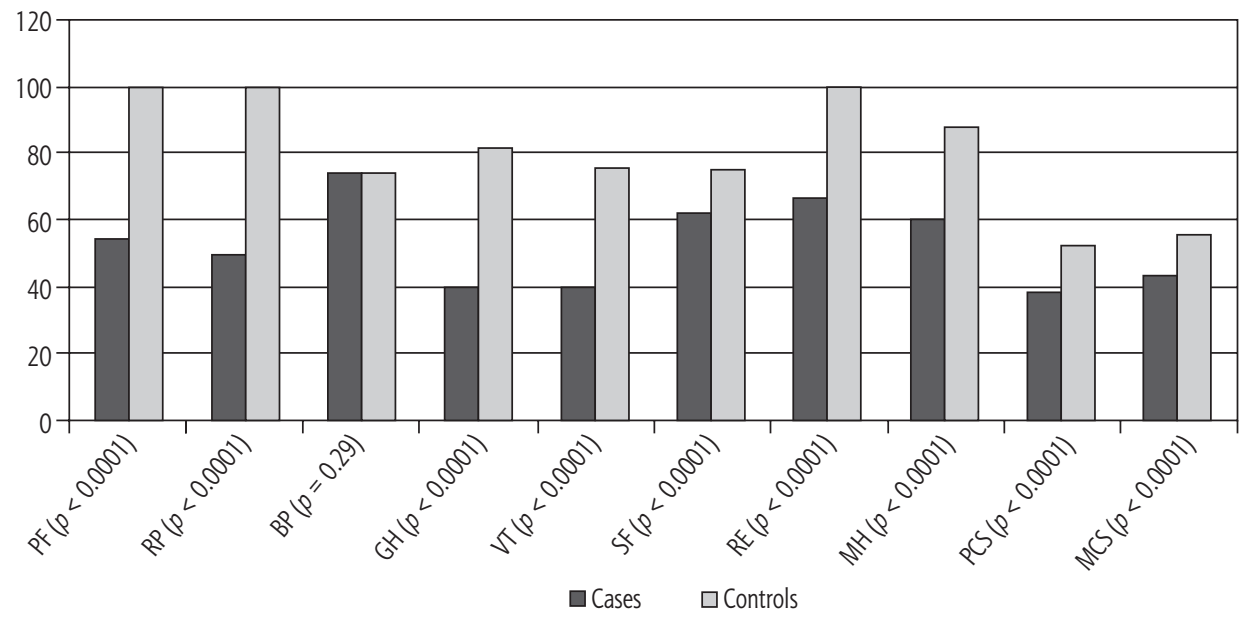

Fig. 1. Comparison of median SF-36 scores among cases and controls

(PCS) and mental health labelled as mental composite score (MCS) using the online calculator. These were computed as the median score with range for comparison. For CLDQ response, likewise, the individual domains were graded on a visual analogue scale of 1 (most impaired) to 7 (least impaired) and further assembled into a single composite overall CLDQ median score.

\section{Statistical analysis}

SPSS version 20 was used. The chi square test was used for proportions, ANOVA for means, an Kruskal-Wallis test for medians. Stepwise linear regression was performed to study the influence of independent variables (CTP score, hepatocellular carcinoma HCC, MELD and complications), on domains of SF-36 and CLDQ while controlling for the effects of other variables. A $p$ value of $<0.05$ was considered as significant for all statistical tests.

\section{Results}

\section{Baseline characteristics}

Amongst the 149 cases and age-gender matched controls, diabetes mellitus in conjunction with or without other co-morbid illness was statistically significantly more frequent among cases $(p<0.0001)$ (Table 1). The median SF-36 score for individual domains (except bodily pain) and composite scores (PCS and MCS) were significantly lower amongst the cases $(p<0.0001)$ (Fig. 1) compared to controls.

There were 44 patients (29.5\%) in CTP A, 49 in CTP B (32.9\%) and 56 in CTP C (37.6\%). Compared to CTP A and $\mathrm{B}$, patients in CTP $\mathrm{C}$ were younger $(48.3 \pm 9.6$ years) (ANOVA $p$ value $<0.005$ ), with a significantly higher median MELD of 24 (range 9 to 41 ) (KW $p<0.0001$ ), and higher complication rates of hepatic encephalopathy $(p<0.005)$ and ascites $(p<0.005)($ Table 2$)$.

\section{Effects of age, co-morbidity, severity of liver disease (CTP scores) and cirrhosis-associated complications on overall and individual domains of SF-36 and CLDQ scores}

\section{By age (Table 3)}

In order to facilitate a meaningful statistical analysis by age, patients were grouped as $\leq 45$ years, 46 to $<55$ years and $\geq 55$ years since $99 \%$ of the cases were distributed between 35 and 65 years. 
Table 2. Baseline demographic and clinical characteristics of cases

\begin{tabular}{|c|c|c|c|c|}
\hline & CTP A & CTP B & CTP C & \multirow{2}{*}{$\begin{array}{l}\text { Chi square/ANOVA*/KW* } \\
\qquad p \text { value }\end{array}$} \\
\hline & №. $(\%)$ & №. (\%) & No. $(\%)$ & \\
\hline Men & $36(81.0)$ & $43(87.8)$ & $47(83.9)$ & NS (0.72) \\
\hline \multirow[t]{2}{*}{ Age (mean $\pm S D$ ) } & $53.3 \pm 7.9$ & $54.0 \pm 9.8$ & $48.3 \pm 9.6$ & $<0.005^{*}$ \\
\hline & & Aetiology & & \\
\hline Alcohol & $10(22.7)$ & $21(42.9)$ & $21(42.9)$ & $<0.01$ \\
\hline Virus (HBV/HCV) & $13(29.5)$ & $9(18.4)$ & $7(12.5)$ & NS \\
\hline Cryptogenic & $20(45.5)$ & $9(18.4)$ & $14(25.0)$ & $<0.001$ \\
\hline \multirow[t]{2}{*}{ Others } & none & $6(12.2)$ & $5(8.9)$ & NS \\
\hline & & Co-morbidity & & \\
\hline Diabetes mellitus & $26(59.1)$ & $31(63.3)$ & $22(39.3)$ & $<0.05$ \\
\hline Arthralgia/back ache/psychiatric disorders & $2(4.6)$ & $15(32.7)$ & $21(37.5)$ & $<0.005$ \\
\hline Diabetes mellitus + other co-morbidity & $17(32.7)$ & $15(30.6)$ & $19(34.0)$ & NS \\
\hline \multirow[t]{2}{*}{ MELD (median; range) } & $11(6-17)$ & $15(6-39)$ & $24(9-41)$ & $<0.0001 * *$ \\
\hline & \multicolumn{3}{|c|}{ Cirrhosis-related complications } & \\
\hline Variceal bleeding & $8(18.2)$ & $9(18.4)$ & $13(23.2)$ & NS \\
\hline Spontaneous bacterial peritonitis & none & $2(4.1)$ & $3(5.3)$ & NS \\
\hline Hepatic encephalopathy & $4(9.1)$ & $6(12.2)$ & 18 (32.1) & $<0.005$ \\
\hline Ascites & 17 (38.6) & $25(51.0)$ & 40 (71.4) & $<0.005$ \\
\hline Acute kidney injury & $3(6.8)$ & $6(12.2)$ & $9(16.1)$ & NS \\
\hline
\end{tabular}

Table 3. Effect of age on SF-36 and CLDQ domains (median; range)

\begin{tabular}{|c|c|c|c|c|}
\hline \multicolumn{5}{|c|}{ Age groups (no. of patients) } \\
\hline & $\leq 45(41)$ & $46-<55(54)$ & $\geq 55$ (49) & $\begin{array}{c}\mathrm{KW} \text { test (Monte Carlo) } \\
p \text { value }\end{array}$ \\
\hline SF-36 Domains & & Median (range) & & \\
\hline PF & $55(10-100)$ & $72.5(0-100)$ & $50(0-100)$ & $<0.05$ \\
\hline RP & $25(0-100)$ & $25(0-100)$ & $50(0-100)$ & NS \\
\hline $\mathrm{BP}$ & $62(0-100)$ & $72(10-100)$ & $84(31-100)$ & $<0.005$ \\
\hline GH & $35(5-90)$ & $42.5(5-92)$ & $45(0-92)$ & NS \\
\hline VT & $35(15-75)$ & $40(15-90)$ & $45(3-90)$ & NS \\
\hline SF & $50(13-100)$ & $62(0-100)$ & $62.5(13-100)$ & NS \\
\hline RE & $66.7(0-100)$ & $67(0-100)$ & $100(0-100)$ & NS \\
\hline $\mathrm{MH}$ & $48(32-94)$ & $58(32-100)$ & $60(36-100)$ & NS \\
\hline PCS & $35(21-56)$ & $41.2(15-62)$ & $41(21-54)$ & NS \\
\hline MCS & $39.7(28-60)$ & $45(24-62)$ & $43.9(23-60)$ & $<0.05$ \\
\hline CLDQ Domains & & Median (range) & & \\
\hline AS & $9.3(0-16)$ & $11.3(0-16)$ & $11.5(0-34)$ & $<0.05$ \\
\hline FA & $17(0-29)$ & $19.6(0-27)$ & $20.1(0-28)$ & NS $(0.3)$ \\
\hline SS & $27(0-35)$ & $28.5(0-35)$ & $27.5(0-35)$ & NS (0.3) \\
\hline$A C$ & $9.7(0-16)$ & $11.2(0-63)$ & $10.5(0-16)$ & NS $(0.5)$ \\
\hline EF & $31.9(0-50)$ & $37.4(0-106)$ & $36.9(0-50)$ & NS (0.07) \\
\hline wo & $22(0-35)$ & $23(0-35)$ & $24(0-35)$ & NS (0.09) \\
\hline Overall CLDQ Score & $118.2(0-180)$ & $134.4(0-199)$ & $133.9(0-179)$ & $<0.05$ \\
\hline
\end{tabular}


Table 4. Effect of diabetes with or without other co-morbidity on SF-36 and CLDQ domains (median, range)

\begin{tabular}{|c|c|c|c|c|}
\hline SF-36 Domains & No co-morbidity (35) & $\begin{array}{l}\text { Diabetes mellitus with or without } \\
\text { other co-morbidity (76) }\end{array}$ & $\begin{array}{l}\text { Co-morbidity other than } \\
\text { diabetes mellitus (34) }\end{array}$ & $\begin{array}{c}\text { Kruskal-Wallis (Monte Carlo) } \\
\quad p \text { value }\end{array}$ \\
\hline PF & $75(25-100)$ & $52.5(0-100)$ & $50(5-90)$ & $<0.01$ \\
\hline $\mathrm{RP}$ & $50(0-100)$ & $37.5(0-100)$ & $25(0-100)$ & $<0.01$ \\
\hline $\mathrm{BP}$ & $91(41-100)$ & $74(31-100)$ & $54(0-100)$ & $<0.0001$ \\
\hline GH & 37 (5-92) & $45(5-90)$ & $30(0-92)$ & NS $(0.2)$ \\
\hline VT & $40(20-75)$ & $40(3-90)$ & $35(15-80)$ & NS $(0.6)$ \\
\hline SF & $75(25-100)$ & $62.3(0-100)$ & $50(13-100)$ & NS (0.07) \\
\hline RE & $100(0-100)$ & $67(0-100)$ & $66.7(0-100)$ & NS $(0.08)$ \\
\hline $\mathrm{MH}$ & $52(32-100)$ & $60(36-96)$ & $52(32-100)$ & NS (0.7) \\
\hline PCS & $44.5(32-55)$ & $38.3(15-62)$ & $34.1(19-54)$ & $<0.0001$ \\
\hline MCS & $42.6(28-61)$ & $43.9(23-66)$ & $42.8(28-61)$ & NS $(0.5)$ \\
\hline $\begin{array}{l}\text { Domains of CLDQ } \\
\text { Scores }\end{array}$ & No co-morbidity (35) & $\begin{array}{l}\text { Diabetes mellitus with or without } \\
\text { other co-morbidity (76) }\end{array}$ & $\begin{array}{l}\text { Co-morbidity other than } \\
\text { diabetes mellitus (34) }\end{array}$ & \\
\hline AS & $10.7(0-34)$ & $11.9(0-16)$ & $9.3(0-16)$ & NS $(0.1)$ \\
\hline FA & $19(0-29)$ & $20(0-28)$ & $17.8(0-29)$ & NS $(0.9)$ \\
\hline SS & $28(0-35)$ & $28(0-35)$ & $27(0-35)$ & NS (0.6) \\
\hline$A C$ & $10.3(0-16)$ & $10.7(0-16)$ & $10.3(0-63)$ & NS $(1.0)$ \\
\hline EF & $33.9(0-68)$ & $36.2(0-106)$ & $33.2(0-50)$ & NS $(0.6)$ \\
\hline WO & $23(0-35)$ & $23(0-35)$ & $24(0-35)$ & NS (0.7) \\
\hline Total CLDQ Score & $129.2(0-180)$ & $131.55(0-199)$ & $119.9(0-180)$ & NS (0.7) \\
\hline
\end{tabular}

Both SF-36 and CLDQ scores were significantly affected in patients below or equal to 45 years. Most patients in this age bracket had a higher CTP score (majority CTP C) and median MELD scores. Cirrhosis-related complications such as hepatic encephalopathy and ascites were also significantly higher (Table 3).

The PF (physical function) domain of SF-36 was significantly lower in patients above 55 years (KW $p<0.05$ ) while BP (bodily pain) was significantly lower in those below 45 years (KW $p<0.005)$. Both these domains of physical components significantly lowered the mental component score (MCS) (KW $p<0.05)$ (Table 3).

With CLDQ scores, the domain of abdominal symptom (AS) in patients below or equal to 45 years was significantly lower at a median of 9.3 compared to 11.3 and 11.5 in the other 2 groups. The overall CLDQ score was also lower in these patients $(p<0.05)$.

\section{By co-morbidity - diabetes mellitus (Table 4)}

For analysis on impact of co-morbidity, patients were categorised as having no co-morbidity, diabetes with or without other co-morbidity and co-morbidity in absence of diabetes.
The median scores of individual domains of PF, RP and BP significantly lowered the composite PCS score in patients who had co-morbidity other than $\mathrm{DM}(\mathrm{KW} p<0.01$ to $<0.0001)$. However, with CLDQ scores, none of the domains or overall CLDQ was affected by co-morbidity (with or without diabetes mellitus).

\section{By severity of liver disease (CTP score) (Table 5)}

In SF-36 and CLDQ, all domains (except AC domain of CLDQ) and the composite scores of both the questionnaires were significantly lower in CTP C patients ( $p$ value range $<0.05$ to $<0.00001$ ).

\section{By cirrhosis-related complications}

None of the cirrhosis-related complications influenced the individual domains or the composite scores of SF-36 and overall CLDQ scores ( $p=$ NS).

Linear regression equations (Table 6) were calculated independently for SF-36 (PCS, MCS) and CLDQ using age, gender, co-morbidity, aetiology and clinical variables such as CTP, MELD score and cirrhosis-related complications as denominators. Age groups and 
Table 5. Effect of severity of liver disease according to CTP score on SF-36 and CLDQ domains (median, range)

\begin{tabular}{|c|c|c|c|c|c|}
\hline & & $A(44)$ & B (49) & $C(56)$ & $\begin{array}{c}\mathrm{KW} \text { test (Monte Carlo) } \\
p \text { value }\end{array}$ \\
\hline \multirow{10}{*}{$\begin{array}{l}\text { SF-36 } \\
\text { Domains }\end{array}$} & PF & $82.5(30-100)$ & $55(5-100)$ & $45(0-100)$ & $<0.0001$ \\
\hline & RP & $53.5(0-100)$ & $50(0-100)$ & $25(0-100)$ & $<0.0001$ \\
\hline & $\mathrm{BP}$ & $74(31-100)$ & $84(10-100)$ & $62(0-100)$ & $<0.05$ \\
\hline & GH & $62(5-92)$ & $35(0-72)$ & $30(5-90)$ & $<0.0001$ \\
\hline & VT & $62.5(20-90)$ & $40(3-65)$ & $35(15-75)$ & $<0.0001$ \\
\hline & SF & $75(25-100)$ & $62.5(13-100)$ & $50(10-100)$ & $<0.001$ \\
\hline & RE & $100(0-100)$ & $100(0-100)$ & $66.7(0-100)$ & $<0.05$ \\
\hline & $\mathrm{MH}$ & $72(36-100)$ & $56(32-76)$ & $48(32-94)$ & $<0.0001$ \\
\hline & PCS & $46(33-59)$ & $38.6(19-53)$ & $34.2(15-62)$ & $<0.0001$ \\
\hline & MCS & $50.7(24-32)$ & $42.7(23-66)$ & $41.7(28-61)$ & $<0.0001$ \\
\hline \multirow{7}{*}{$\begin{array}{l}\text { Domains of } \\
\text { CLDQ Scores }\end{array}$} & AS & $14.2(0-11.3)$ & $11.3(0-16)$ & $9.3(0-34)$ & $<0.00001$ \\
\hline & FA & $23.4(0-29)$ & $19(0-27)$ & $17.7(0-26)$ & $<0.01$ \\
\hline & SS & $31(0-35)$ & $28(0-34)$ & $27(0-34)$ & $<0.0001$ \\
\hline & $A C$ & $10.3(0-16)$ & $11.3(0-15)$ & $10.3(0-63)$ & NS (0.1) \\
\hline & $\mathrm{EF}$ & $41.9(0-50)$ & $34.8(0-45)$ & $32.7(0-106)$ & $<0.0001$ \\
\hline & WO & $26(0-35)$ & $23(0-31)$ & $22.5(0-31)$ & $<0.05$ \\
\hline & Total CLDQ score & $147.7(0-180)$ & $130.6(0-163)$ & $120.5(0-199)$ & $<0.001$ \\
\hline
\end{tabular}

Table 6. Linear regression co-efficient for PCS, MCS and CLDQ using independent demographic and clinical variables

\begin{tabular}{|c|c|c|c|c|c|c|c|c|c|c|c|c|}
\hline & \multicolumn{4}{|c|}{ PCS } & \multicolumn{4}{|c|}{ MCS } & \multicolumn{4}{|c|}{ CLDQ } \\
\hline & \multirow{2}{*}{$\begin{array}{c}\text { Std } \\
\text { Coeff } \beta\end{array}$} & \multicolumn{2}{|c|}{$95 \% \mathrm{Cl}$ for $\beta$} & \multirow[t]{2}{*}{ Sig. } & \multirow{2}{*}{$\begin{array}{c}\text { Std } \\
\text { Coeff } \beta\end{array}$} & \multicolumn{2}{|c|}{$95 \% \mathrm{Cl}$ for $\beta$} & \multirow[t]{2}{*}{ Sig. } & \multirow{2}{*}{$\begin{array}{c}\text { Std } \\
\text { Coeff } \beta\end{array}$} & \multicolumn{2}{|c|}{$95 \% \mathrm{Cl}$ for $\beta$} & \multirow[t]{2}{*}{$p$ value } \\
\hline & & lower & upper & & & lower & upper & & & lower & upper & \\
\hline Constant & & 45.8 & 55.2 & $<0.0001$ & & 48.1 & 57.4 & $<0.0001$ & 157.8 & 142.6 & 173.1 & $<0.0001$ \\
\hline Age & -0.1 & -2.8 & 0.06 & 0.06 & 0.16 & 0.7 & 3.5 & $<0.005$ & 0.06 & -4.1 & 7.8 & NS \\
\hline Sex & 0.0 & -2.5 & 2.7 & 1.0 & -0.03 & -3.3 & 1.8 & NS & 0.08 & -5.0 & 15.2 & NS \\
\hline Diabetes & -0.02 & -2.5 & 1.63 & 0.7 & -0.1 & -3.8 & 0.9 & NS & -0.03 & -8.6 & 6.2 & NS \\
\hline Alcohol & -0.129 & -5.6 & 0.02 & 0.05 & 0.04 & -1.9 & 3.5 & NS & -0.13 & -11.8 & 3.5 & NS \\
\hline СTP & 0.04 & -0.5 & 1.0 & 0.5 & 0.01 & -0.7 & 0.8 & 0.9 & -0.36 & -16.4 & -3.5 & $<0.0001$ \\
\hline MELD & 0.44 & -0.6 & -0.3 & $<0.0001$ & -0.39 & -0.5 & -0.2 & $<0.0001$ & -0.23 & -1.2 & -0.03 & $<0.05$ \\
\hline VB & -0.02 & -3.5 & 3.0 & 0.9 & -0.09 & -5.8 & 0.3 & 0.08 & 0.09 & -3.8 & 13.8 & NS \\
\hline SBP & 0.04 & -5.1 & 12.9 & 0.4 & -0.04 & -12.1 & 5.2 & 0.4 & -0.01 & -20.7 & 19.5 & NS \\
\hline $\mathrm{HE}$ & 0.1 & -6.2 & 0.03 & $<0.05$ & -0.06 & -4.8 & 1.2 & 0.2 & 0.02 & -7.4 & 9.8 & NS \\
\hline Ascites & 0.02 & -3.0 & 2.3 & 0.8 & -0.08 & -4.1 & 0.9 & 0.2 & 0.2 & -0.4 & 14.7 & NS \\
\hline AKI & -0.08 & -7.8 & 0.6 & 0.09 & -0.03 & -5.0 & 3.1 & 0.6 & -0.08 & -18.3 & 6.2 & NS \\
\hline
\end{tabular}

alcohol did not decrease the PCS score significantly although the regression coefficient was significant. MELD had a direct and significant association with overall PCS, MCS and CLDQ. For every unit increase of MELD, PCS decreased by $0.44(p<0.0001)$, MCS by $0.39(p<0.0001)$ and CLDQ by $0.24(p<0.05)$. CTP score altered the overall CLDQ score.
Summarising our observations, age below 45 years influenced the composite MCS and overall CLDQ scores. CTP severity and high MELD value had an effect on composite scores of PCS and MCS as well as CLDQ. Diabetes as a co-morbidity and cirrhosis-related complications did not affect the individual or composite domains of the 2 QOL scores. However, 
co-morbidity other than diabetes, e.g. hypertension, hypothyroidism, arthralgia, low back ache, etc., significantly reduced the composite PCS scores.

\section{Discussion}

Our study has addressed the QOL in patients with liver cirrhosis with reference to specific domains of SF-36 and CLDQ questionnaires with respect to age, diabetes mellitus, severity of liver disease (CTP and MELD scores) and cirrhosis-related complications. Our patients had significantly lower SF-36 HRQOL scores compared to the healthy population $(p<0.0001)$ for all domains. Diabetes mellitus with or without other co-morbid illness was significantly higher amongst cases compared to controls. However, it did not influence the domains of CLDQ scores. On the other hand, non-diabetics with other co-morbid states such as arthralgia, fatigue, and psychiatric ailments, influenced the individual domains of physical function and role physical that significantly lowered the PCS with no effect on CLDQ. Sobhonslidsuk et al. [8] observed that advanced age, female gender and lower socioeconomic status reduced QOL.

David et al. [9] observed that type 2 diabetes significantly impaired MCS and PCS in non-alcoholic fatty liver disease patients. Hauser et al. [10] in their series, reported that psychiatric conditions and systemic co-morbidity independently lowered the overall CLDQ and PCS scores. Cardiovascular co-morbidity affected the individual domains of SF-36 in their series. Gutteling et al. [11] using the Liver Disease Symptom Index and SF-6D questionnaire reported poorer HRQOL that was influenced by severity of liver disease, anorexia, fatigue, depression and joint pain.

Age as a determinant has not been studied extensively as an influencing factor on SF-36 and CLDQ. In our study, a significant proportion of patients belonged to CTP C with a median age of 48 years with high rates of complications such as refractory ascites and hepatic encephalopathy. Patients $\leq 45$ years in our study had a significantly lower score for bodily pain $(p<0.005)$ and lower physical function $(p<0.05)$ which resulted in significant reduction in overall MCS. Farivar et al. [12] made a critical observation in their study and stated that higher mental health scores drive the PCS down while higher physical function drives the MCS down. This was also the observation in our study.

Severity of liver disease is known to influence HRQOL $[2,8,10,11,13,14]$. In our study, CTP C classification and younger age, i.e. around 45 years, significantly affected QOL in our patients. These were the patients who also had higher median MELD scores, and higher rates of complications of hepatic encephalopathy and ascites. Symptoms such as fatigue, tense ascites and muscle cramps are associated with poor QOL [2]. Zuberi et al. [15] found lower CLDQ scores in patients with CTP C.

Despite the fact that a significant proportion of our patients belonged to either CTP B or CTP C, cirrhosis-related complications per se did not according to univariate analysis affect the two QOL scores. However, in linear regression co-efficient analysis, hepatic encephalopathy influenced PCS. This observation needs validation with a large sample size. Overt hepatic encephalopathy has been reported to significantly affect PCS [16]. Marchesini et al. [2] observed ascites to be an independent predictor of poor HRQOL in three SF domains, namely bodily pain, general and mental health. Gao et al. [13] in stepwise linear regression analysis found that PCS and MCS were affected by factors such as age, severity of liver disease and cirrhosis-related complications.

In conclusion, patients with cirrhosis do worse in generic (SF36) and disease-specific questionnaires (CLD-Q) compared to controls. QOL in patients with liver cirrhosis is affected by disease severity as evidenced by MELD or CTP scores, but not by presence of diabetes. Physical function is worse at age $>55$ years and bodily pain more frequent at age $<45$ years among cirrhotics. Limitations of the study: fewer cases for comparison between the CTP scores. An in depth study is required to address the effects of individual co-morbid states other than diabetes on QOL. This was not possible in this study as the number of cases with specific co-morbidity, e.g. arthralgia, psychiatric illness, etc, was too low to reach statistical significance.

\section{Disclosure}

Authors report no conflict of interest.

\section{References}

1. Younossi ZM, Boparai N, McCormick M, et al. Assessment of utilities and health-related quality of life in patients with chronic liver disease. Am J Gastroenterol 2001; 96: 579-583.

2. Marchesini G, Bianchi G, Amodio P, et al. Factors associated with poor health-related quality of life of patients with cirrhosis. Gastroenterology 2001; 120: 170-178.

3. Younossi ZM, Boparai N, Price LL, et al. Health-related quality of life of patients in chronic liver disease :the impact of type and severity of liver disease. Am J Gastroenterol 2001; 96: 21992205.

4. Sobhonslidsuk A, Silpakit C, Kongsakon R, et al. Chronic liver disease questionnaire translation and validation in Thais. World J Gastroenterol 2004; 10: 1954-1957.

5. Ray I, Dutta D, Basu P, de BK. Quality of life assessment of patients with chronic liver disease in eastern India using a Bengali 
translation chronic liver disease questionnaire. Indian J Gastroenterol 2010; 29: 187-195.

6. Younossi ZM, Guyatt G, Kiwi M, et al. Development of a disease specific questionnaire to measure health related quality of life in patients with chronic liver disease. Gut 1999; 45: 295-300.

7. Matteoni CA, Younossi ZM, Gramlich T, et al. Nonalcoholic fatty liver disease: A spectrum of clinical and pathological severity. Gastroenterology 1999; 116: 1413-1419.

8. Sobhonslidsuk A, Silpakit C, Kongsakon R, et al. Factors influencing health-related quality of life in chronic liver disease. World J Gastroenterol 2006; 12: 7786-7791.

9. David K, Kowdley KV, Unalp A, et al. Quality of life in adults with nonalcoholic fatty liver disease: baseline data from the nonalcoholic steatohepatitis clinical research network. Hepatology 2009; 49: 1904-1912.

10. Hauser W, Holtman G, Grandi D. Determinants of health related quality of life in patients with chronic liver disease. Clin Gastroenterol Hepatol 2004; 2: 157-163.

11. Gutteling JJ, deMan RA, van der Plas SM, et al. Determinants of quality of life in chronic liver patients. Aliment Pharmacol Ther 2006; 23: 1629-1635.

12. Fariver SS, Cunningham WE, Hays RD. Correlated physical and mental health summary scores for the SF-36 and SF-12 Health Survey, V.1. Health Qual Life Outcomes 2007; 5: 54.

13. Gao R, Gao F, Li G, Hao JY. Health related quality of life in Chinese patients with chronic liver disease. Gastroenterol Res Prac 2012; 2012; 516140 .

14. Hauser W, Holtman G, Grandi D. Determinants of health related quality of life in patients with chronic liver disease. Clin Gastroenterol Hepatol 2004; 2: 157-163.

15. Zuberi BF, Memom AR, Afsar S, et al. Correlation of quality of life in patients of cirrhosis of liver with etiology and disease severity using disease specific quality of life questionnaire. J Ayub Med Coll Abbottabad 2007; 19: 7-11.

16. Arguedas MR, DeLawrence TG, McGuire BM. Influence of hepatic encephalopathy on health-related quality of life in patients with cirrhosis. Dig Dis Sci 2003; 48: 1622-1626. 